\title{
Neuro Rehabilitation Care during COVID Times and Beyond
}

\section{Vijay Janagama*}

Director, SuVitas Holistic Health Care Pvt Ltd, India

*Corresponding Author: Vijay Janagama, Director, SuVitas Holistic Health Care Pvt Ltd, India.
Received: September 14, 2021

Published: October 28, 2021

(C) All rights are reserved by Vijay Janagama.
COVID-19 has created unprecedented stress across all sectors, including healthcare. However, we have seen some success points during this long and ongoing battle. Early in the pandemic itself we were able to diagnose the disease, knew preventive tactics using face mask, hand hygiene and physical distance. We could also make vaccines available in the shortest time in the history of vaccines. Many shortcomings were evident too. Definitive treatment approaches are yet to get consensus from medical community, faith in efficacy and safety of vaccination, timing of relaxing COVID norms are a few that fall into diverse opinions. While all these issues are staged around COVID, one critical point that still awaits definitive answer is the concrete approach on managing non COVID services across health care spectrum. One such pivotal area is continuing Rehabilitation Care during and beyond COVID times. This applies to every condition that can benefit from rehabilitation. Specialities like Neurology and Neurosurgery need particular attention as these diseases impact all aspects of society if not handled well.

Neuro Rehabilitation is a multi-disciplinary dynamic set of therapeutic interventions offered to aide in physical, functional and emotional recovery after neurological disease/injury. Conditions that benefit from Neuro Rehabilitation include: cardiovascular disorders like Stroke, Aneurysm, Hematoma, Neuromuscular disorders like Guillain Barre Syndrome, Myasthenia Gravis, Injuries of brain, head or spinal cord, infections like meningitis, encephalitis, degenerative disorders like Alzheimer's, Parkinson's, Multiple Sclerosis, Amyotrophic Sclerosis, Functional Disorders like headache, epilepsy and neuralgia. While this is not a complete list, this would reflect the long list of conditions that could be benefited from neuro rehabilitation. As with many Non COVID services, it was challenging to maintain the continuity of neuro rehabilitation during the pandemic. Achieving maximum possible functional and emotional recovery, preventing complications and recurrences are known benefits of neuro rehabilitation. Understanding the value of early and ongoing rehabilitation, we need to mitigate the multiple moving options to ensure continuity of neuro rehabilitation. Keeping the traditional primary care as a focus, it is time to adopt and adapt different models of health care delivery to ensure timely and continued neuro rehabilitation.

Tertiary and quaternary care will have to be prepared, optimized and preserved for criterion specific admissions. In established health care models, Long Term Acute Care models have provided leeway to Intensive Care Units utility. Transition Care Units like Inpatient Rehabilitation Facilities and Skilled Nursing Care facilities have played a pivotal role in improving recovery and reducing recurrences, cost and complications. During times of restrictive travel, tele medicine has realized instant access to medical fraternity and pandemic expedited adoption of technology. Home Health Care and Assisted Living facilities went through initial uncertainty on continuity, yet are now resuming to serve routine demands. Innovative models like mobile rehabilitation units can pave way for enhanced access to rehabilitation care. Requirements can be complex in neuro rehabilitation ranging from skilled nursing care like tracheostomy, deep wound care, physiotherapy for completely bed ridden conditions and cognitive therapy for semi-comatose cases. It is essential to have a multidisciplinary team comprising of doctor, nurse, dietician, physiotherapist, occupational therapist, speech 
therapist, psychologist, and coordinator to ensure the prompt and smooth coordination of all interventions and their implementations. In evolving health care models, where all these spectrum of service deliveries are not well established, initiating a hybrid model might be a good start to ensure optimal utilization of scarce resources.

As sequential COVID waves take their turn, it is pivotal now to proactively plan for treating acute COVID phase and also preventing short and long-term complications of the disease. It is established that COVID is more than a respiratory disease and it can have neurological manifestations as well. Continuing Non COVID medical emergencies, long term illness management and rehabilitation in specialities like neurology, neurosurgery, oncology, cardiovascular diseases have to be proactively planned for prompt implementation, as they themselves are an independent threat and also can complicate COVID management. If implemented well, models adopted during the stressful pandemic time can leave a lasting impact on disease management of COVID and beyond.

Volume 4 Issue 11 November 2021

CAll rights are reserved by Vijay Janagama.

Citation: Vijay Janagama. "Neuro Rehabilitation Care during COVID Times and Beyond". Acta Scientific Neurology 4.11 (2021): 55-56. 